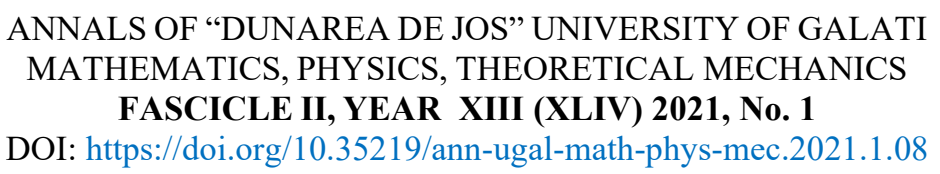

\title{
Color space influence on ANN skin lesion classification using statistics texture feature
}

\author{
Felicia Anisoara Damian ${ }^{1,3}$, Simona Moldovanu ${ }^{2,3}$, Luminita Moraru ${ }^{1,3, *}$
}

\author{
I"Dunarea de Jos" University of Galati, Faculty of Sciences and Environment, 111 Domneasca street, 800201, \\ Galati, Romania \\ 2"Dunarea de Jos" University of Galati, Faculty of Automation, Computers Sciences, Electronics and Electrical \\ Engineering, Department of Computer Science and Information Technology, 2 Stiintei street, 800210, Galati, \\ Romania \\ 3"Dunarea de Jos" University of Galati, The Modelling \& Simulation Laboratory Galati, 47 Domneasca street, \\ 800008 Galati, Romania \\ *Corresponding author: Luminita.Moraru@ugal.ro
}

\begin{abstract}
This study aims to investigate the ability of an artificial neural network to differentiate between malign and benign skin lesions based on two statistics terms and for RGB (R red, G green, B blue) and YIQ (Y luminance, and I and Q chromatic differences) color spaces. The targeted statistics texture features are skewness (S) and kurtosis $(\mathrm{K})$ which are extracted from the histograms of each color channel corresponding to the color spaces and for the two classes of lesions: nevi and melanomas. The extracted data is used to train the Feed-Forward Back Propagation Networks (FFBPNs). The number of neurons in the hidden layer varies: it can be 8, 16, 24, or 32 . The results indicate skewness features computed for the red channel in the RGB color space as the best choice to reach the goal of our study. The reported result shows the advantages of monochrome channels representation for skin lesions diagnosis.
\end{abstract}

Keywords: RGB, YIQ, skewness, kurtosis, Feed-Forward Back Propagation Network (FFBPN)

\section{INTRODUCTION}

Although melanoma accounts for only about $1 \%$ of skin cancers ${ }^{1}$, it is the type of cancer that causes the majority of deaths. Melanoma occurs when pigment-producing cells called melanocytes multiply uncontrollably. If melanoma is detected as early as possible it can be completely treated.

Pixels in digital images can be used to analyze, classify, and identify objects in images. Within the YIQ color space, the luminance is represented as a grayscale signal. The I (hue) and Q (saturation) components indicate the chromatic differences. The value " 0 " corresponds to the absence of the component, while "1" means a complete saturation of the component. The RGB color space focuses on the color information provided by statistical parameters additively computed by using the intensity values of the monochrome $\mathrm{R}, \mathrm{G}$, and $\mathrm{B}$ channels [1]. The image histogram is a graphical representation of the intensity of the pixels and allows one to compute various statistics features. Skewness (S) and kurtosis (K) provide information about the tails of the distribution of pixel intensities [2]. Thus, $\mathrm{S}$ characterizes the relative size of the two tails while $\mathrm{K}$ estimates the combination of the two tails.

${ }^{1}$ https://www.cancer.org/cancer/melanoma-skin-cancer/about/key-statistics.html 
Kwok et al. [3], studied the effect of different color models on the performance of the color image segmentation. They investigated the appropriateness of the choice of color model for aerial images, as YIQ, YUV, I1I2I3, HIS, and HSV and proposed a segmentation procedure based on maximizing the content of the information entropy calculated from the probability distribution. The highest information content was obtained from the "R" and "Y" color channels for RGB and YIQ color models, respectively. Khan et al. [4], performed the color image segmentation of skin lesions by using the Fuzzy C-means clustering algorithm. In this approach, 50 color images were transformed into different samples for RGB, normalized RGB, YIQ, and I1I2I3 color models. The images were decomposed into a certain number of homogeneous regions based on the similarity comparison with the color models. The best results were obtained from the Q channel of the YIQ color space and the I3 channel of the I1I2I3 color space, as they are robust to uneven lighting. Damian et. al [5] proposed a simple method for differentiating between skin lesions by using a single-color channel; moreover, their method is based on features derived from gray scale images extracted from individual monochrome channels. The color histograms were built for red, green, and blue channels and were analyzed using the histogram spread (HS) function. The reported results suggested that the pixel values for the red channel are strongly related to the type of skin lesion. Bakheet and Al-Hamadi [6] proposed a CAD method for melanoma detection, using an improved multicast driven neural network based on the Levenberg-Marquandt algorithm. Each image is denoised and some operations for image quality improving were performed. The feature extraction was performed on segmented images. These features were normalized and were used as input for a multilevel neural network trained to classify each skin lesion. The proposed method was tested and validated using cross-validation and provided high levels of accuracy. Kanimozhi and Murthi [7] handled the images by having minimized the background influence and detected the edge of the skin lesions. The well-known geometric features such as asymmetry, edge, color, and diameter were extracted. By using the ABCD rule, they used a backpropagation neural network for skin lesions classification. They reported an accuracy of $96.9 \%$.

In the present study, we propose a method to differentiate between two categories of skin lesions i.e., nevi and melanomas, with the final goal being to determine the best color space in which we would be able to perform this task by using two statistics features. The skewness and kurtosis statistics features are extracted from the color histogram of dermatoscopic images in the RGB and YIQ color spaces.

\section{METHODS}

For an image, the information embedded in each color space is potentially different as the light and color intensity information change from color space to color space. Similar findings apply to each mono-channel in a color space. However, it may happen that different channels in the color spaces include similar or almost identical information. Thus, the efficiency of the statistics features retrieved from each channel in the color spaces for skin lesion classification is investigated using an ANN tool. To suppress the color variations of the skin lesions and to reduce the effect of various skin color variations, YIQ is linearly transformed from the RGB color space, as follows [8]:

$$
\begin{aligned}
& \mathrm{Y}=0.299 R+0.587 \mathrm{G}+0.114 \mathrm{~B} \\
& \mathrm{I}=0.596 \mathrm{R}-0.274 \mathrm{G}-0.322 \mathrm{~B} \\
& \mathrm{Q}=0.212 \mathrm{R}-0.523 \mathrm{G}+0.311 \mathrm{~B}
\end{aligned}
$$

The $\mathrm{S}$ and $\mathrm{K}$ features are extracted from the histograms of each color channel corresponding to the color spaces. $\mathrm{S}$ is a measure of the histogram asymmetry of image-specific gray levels [9]. $\mathrm{K}$ is the degree of vaulting of the gray level histogram and is affected by the outliers in a sample [10]. For univariate data $\mathrm{Y} 1, \mathrm{Y} 2, \ldots, \mathrm{YN}, \mathrm{S}$, and $\mathrm{K}$ are given by: 


$$
\begin{aligned}
& S=\frac{1}{N} \frac{\sum_{i=1}^{N}\left(Y_{i}-\vec{Y}\right)^{3}}{s^{3}} \\
& K=\frac{1}{N} \frac{\sum_{i=1}^{N}\left(Y_{i}-\vec{Y}\right)^{4}}{s 4}
\end{aligned}
$$
histogram.

Here, $\vec{Y}$ is the mean, s is the standard deviation, and $\mathrm{N}$ is the number of gray levels in the

ANN consists of an input layer, an output layer, and a hidden layer. Input nodes take information in numerical form. The information is presented as activation values, where each node is assigned a number, such that the higher the number, the higher the activation is. In a case of a feedforward network, the information is then transmitted forward throughout the network [11, 12]. The activation value is transmitted from node to node but weighted in a certain manner. The activation values are reviewed at each node. The activation circulates through the network, through hidden layers, until it reaches the output nodes. The difference between the predicted value and the real value (error) will be backpropagated throughout the network. The transfer function converts the input signals into output signals. In the proposed method the sigmoid transfer function is used [13].

Each training network is a pair of the form $\{\vec{X}, \vec{Y}\}$, where $\mathrm{X}$ is a vector comprising of the input data values and $\mathrm{Y}$ is a vector of target values (desired values). The architecture of the proposed model is displayed in Fig. 1.

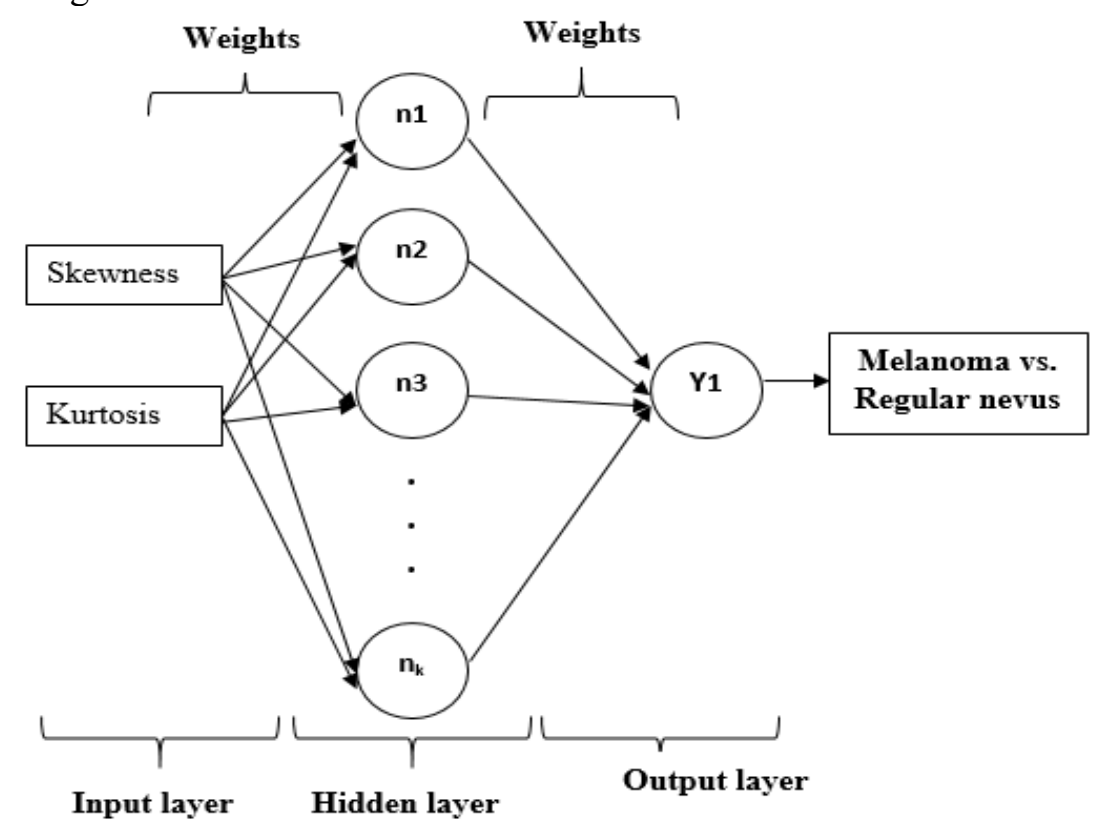

Fig. 1. Feed Forward Back Propagation Network FFBPN architecture with $n=8,16,24$ and 32 neurons.

The proposed FFBNP model used as input the extracted $\mathrm{S}$ and $\mathrm{K}$ image features from each color channel and then, classify the output as 0 and 1 , where 0 is assigned for nevus and 1 for melanoma.

The algorithm of the proposed method is presented in Fig. 2. 


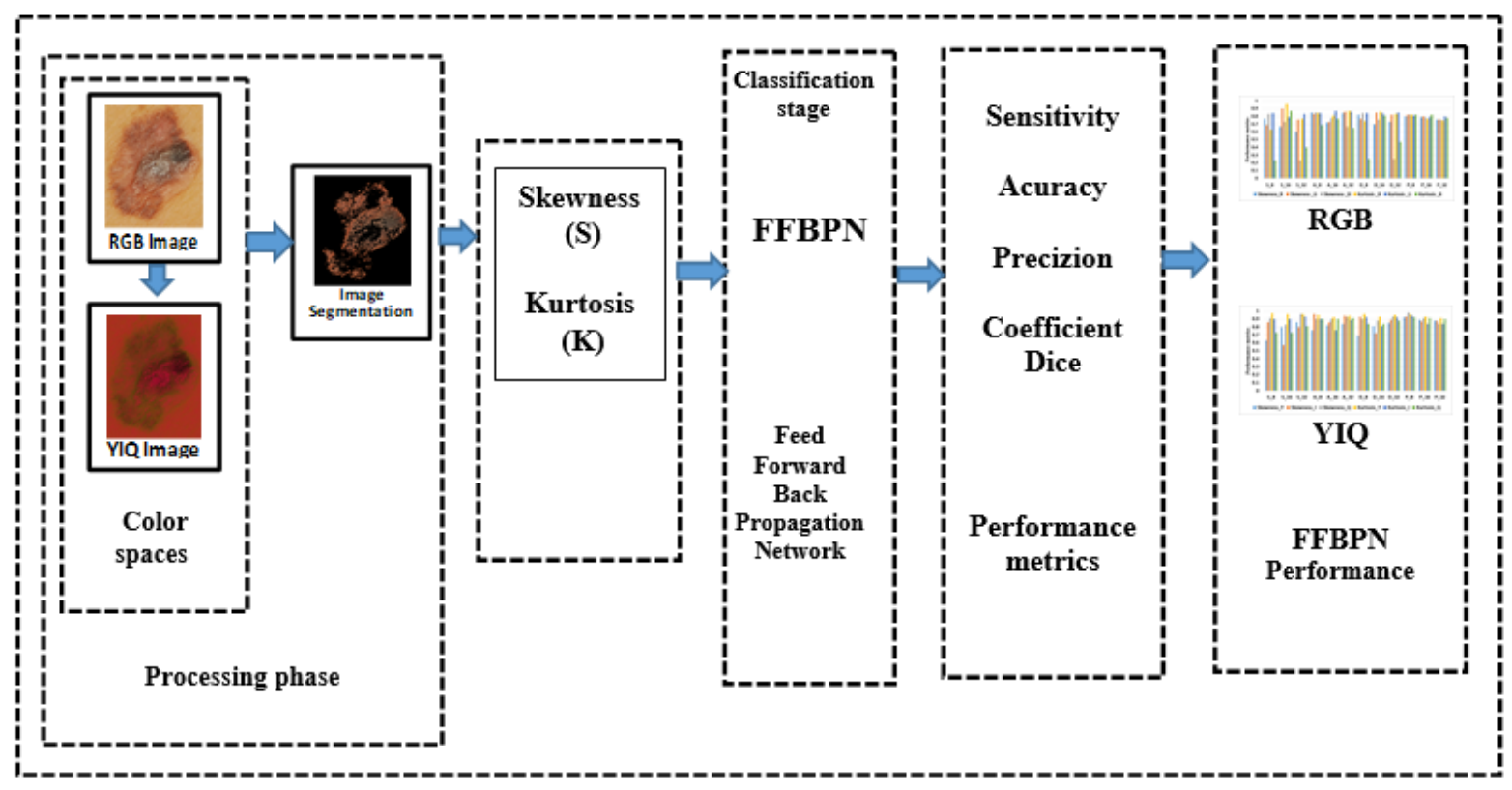

Fig. 2. Pipeline scheme

To evaluate the performance of the proposed method, the same evaluation metrics largely used in the literature are computed from the confusion matrix.

$$
\begin{aligned}
& \text { Sensitivity }(\mathrm{SE})=\frac{\mathrm{TP}}{\mathrm{TP}+\mathrm{FN}} \\
& \text { Accuracy }(\mathrm{ACC})=\frac{\mathrm{TP}+\mathrm{TN}}{\mathrm{TP}+\mathrm{TN}+\mathrm{FP}+\mathrm{FN}} \\
& \operatorname{Precision}(\mathrm{P})=\frac{\mathrm{TP}}{\mathrm{TP}+\mathrm{FP}}
\end{aligned}
$$

Dice coefficient $(D)=\frac{2 T P}{2 T P+F P+F N}$

where TP is true positives, FP false positives, TN true negatives, and FN false negatives.

\section{Image segmentation}

The colour histogram is a method for describing the colour content of an image: it counts the number of occurrences of each colour in an image [14]. For a given RGB image, let L be the intensity levels in the range $[0,1,2, \ldots, \mathrm{L}-1]$. Then, the probability distribution can be defined as:

$$
\mathrm{p}_{\mathrm{i}}^{\mathrm{C}}=\frac{\mathrm{h}_{\mathrm{i}}^{\mathrm{C}}}{\mathrm{N}}, \sum_{\mathrm{i}=0}^{\mathrm{L}-1} \mathrm{p}_{\mathrm{i}}^{\mathrm{C}}=1
$$


where $\mathrm{i}$ is a specific intensity level in the range $\{0 \leq \mathrm{i} \leq \mathrm{L}-1\}$ for the colour component $\mathrm{C}=$ $\{R, G, B\}, N$ is the total number of pixels in the image and $h_{i}^{C}$ is the number of pixels for the corresponding intensity level $\mathrm{i}$ in component $\mathrm{C}$ [15]. The histogram separates the foreground from the background. The minimum value between these two peaks is the optimum threshold used in image segmentation.

\section{EXPERIMENTAL DATABASE AND ALGORITHM}

In this study, the MATLAB R2017a programming environment was used to develop the FFBPN model. The experimental hardware environment was a computer with the following specifications: Processor: Inter (R) Core (TM) i3-4030U, $1.9 \mathrm{GHz}$, Installed memory (RAM): $4 \mathrm{~Gb}$, System type: 64-bit Operating System, x64-based processor. The object of study contains 140 color digital images (70 melanomas and 70 nevi), which were acquired from the digital archive of the Department of Dermatology of the University Medical Center of Groningen (UMCG)2. They were used for the development and testing of the MED-NODE system for skin cancer detection in RGB color digital images. The proposed algorithm implements the initial data step by step - this refers to color digital images encoded in the RGB color spaces, which are then transformed into the YIQ color spaces. Further, the segmentation based on the color histogram of digital images was performed to calculate the $\mathrm{S}$ and $\mathrm{K}$ features. The algorithm's output is represented by its values.

Experiments performed to investigate the utility of color spaces in the diagnosis of skin lesions include the following steps:

\{Step 1\} Separate images into color channels;

\{Step 2\} Image segmentation;

\{Step 2\} Build the histogram of each color channel for RGB and YIQ systems;

\{Step 3 For each channel, compute the $\mathrm{S}$ and $\mathrm{K}$ features;

\{Step 4 \} Repeat steps 1 through 3, for the entire image database;

\{Step 5\} FFBPN training for each channel;

\{Step 6\} Analyze the results in terms of performance metrics: sensitivity, accuracy, Dice coefficient and precision, for each color channel.

\section{RESULTS AND DISCUSSION}

The color histogram characterizes the nature of the skin lesion depending on the probability of occurrence of the intensities of the color pixels in a channel. The graphical representation of the intensity of color pixels for melanomas for the two color spaces is shown in Figures 3 and 4, for melanoma and nevi, respectively. For melanoma, high peaks were observed for both color spaces. For nevi, flat peaks were observed for the RGB color space and slightly high for the YIQ color space. Thus, the identified variations in the color histogram could be used to determine the nature of the skin lesion.

From the histograms, the $\mathrm{S}$ and $\mathrm{K}$ features are extracted [16]. An analysis of the asymmetric distribution of the intensities of the pixels for a lesion compared to the average of each color channel is relevant in this case.

\footnotetext{
2 http://www.cs.rug.nl/ imaging/databases/melanoma naevi/
} 


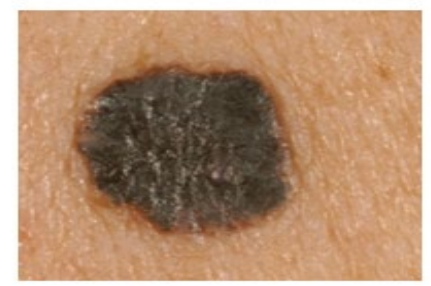

(a)

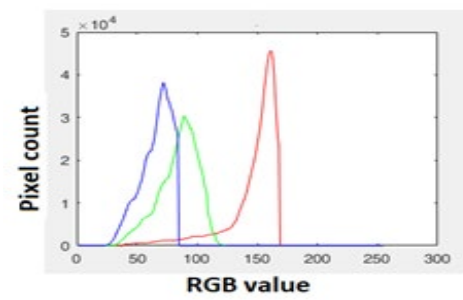

(c)

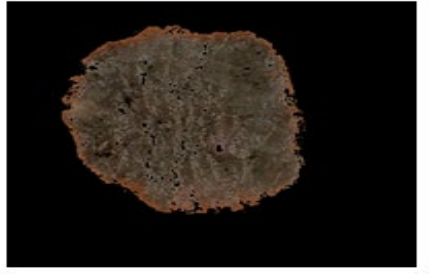

(b)

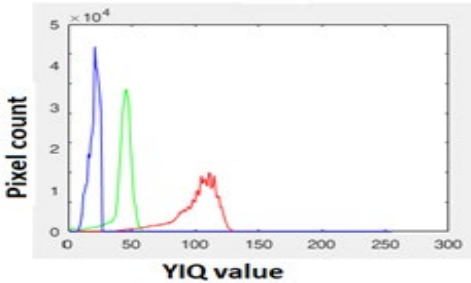

(d)

Fig.3. Color histogram of melanoma

(a) RGB image; (b) Image segmentation; (c) Histogram of RGB channels; (d) Histogram of YIQ channels

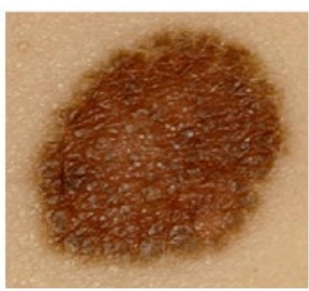

(a)

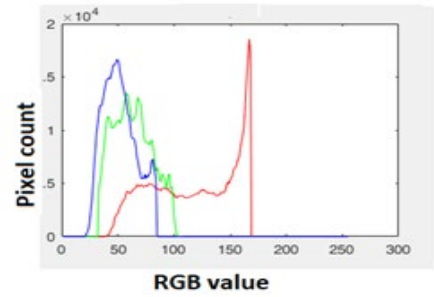

(c)

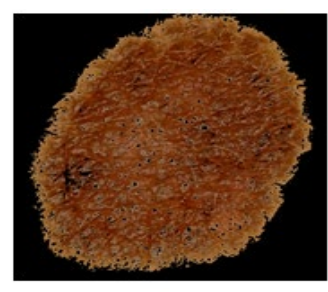

(b)

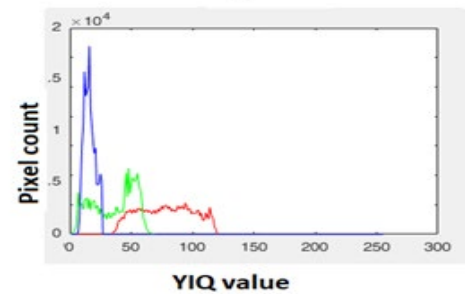

(d)

Fig. 4. Color histogram of nevi

(a) RGB image; (b) Image segmentation; (c) Histogram of RGB channels; (d) Histogram of YIQ channels

The input data for FFBNP are the $\mathrm{S}$ and $\mathrm{K}$ features which are retrieved from the histogram of each color channel corresponding to the RGB and YIQ color systems. The FFBPN has also been trained using $\mathrm{S}$ and $\mathrm{K}$ data computed for nevi and melanomas, for all available color channels. By default, the training set was $70 \%$ from the dataset, the test and validation sets were $15 \%$ each. The performance metrics were calculated for each FFBPN architecture (i.e., 8, 16, 24, and 32 hidden neurons). The values of the performance metrics vary between 0 and 1 . Figure 5 presents the total classification accuracy of the FFBPN, for all performance metrics and the number of hidden neurons. By using the results of this analysis, the number of neurons in the hidden layer is varied from 8 to 32 . This number directly influences the classification accuracy. 


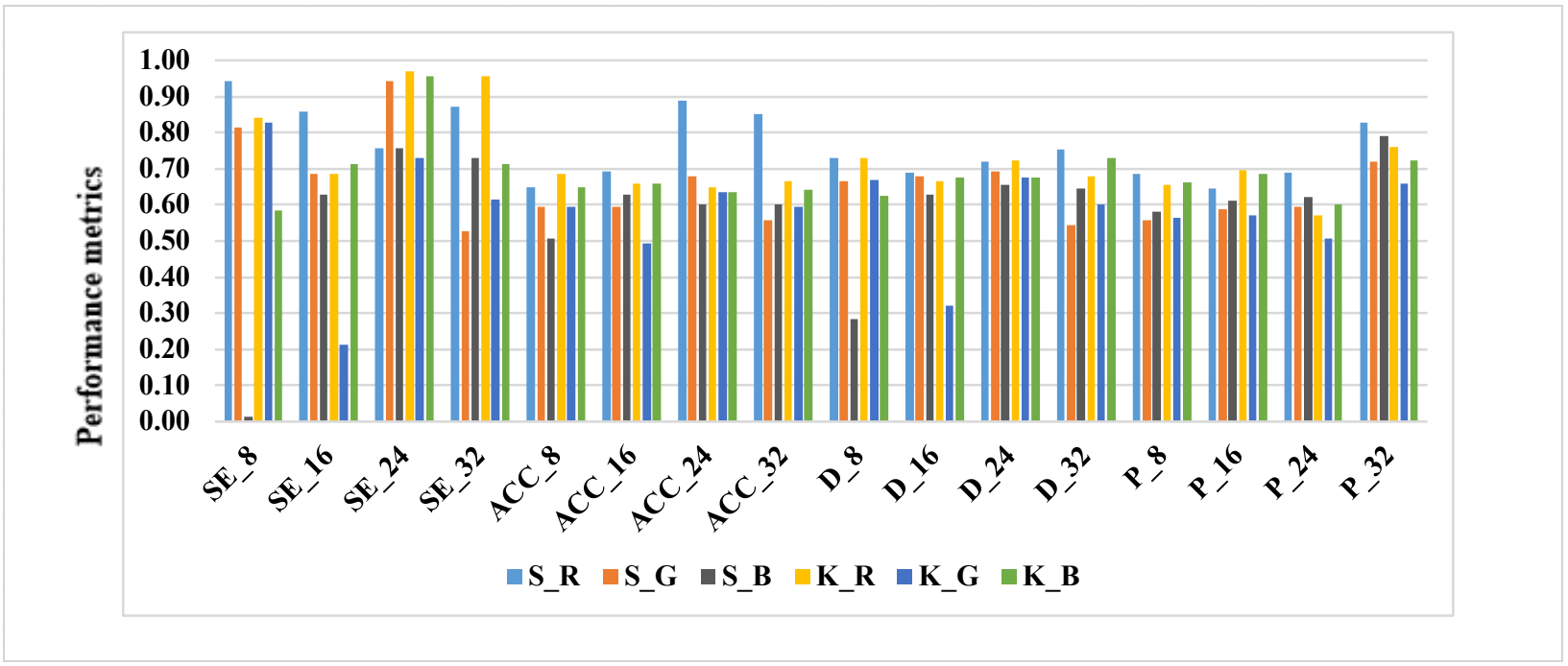

(a)

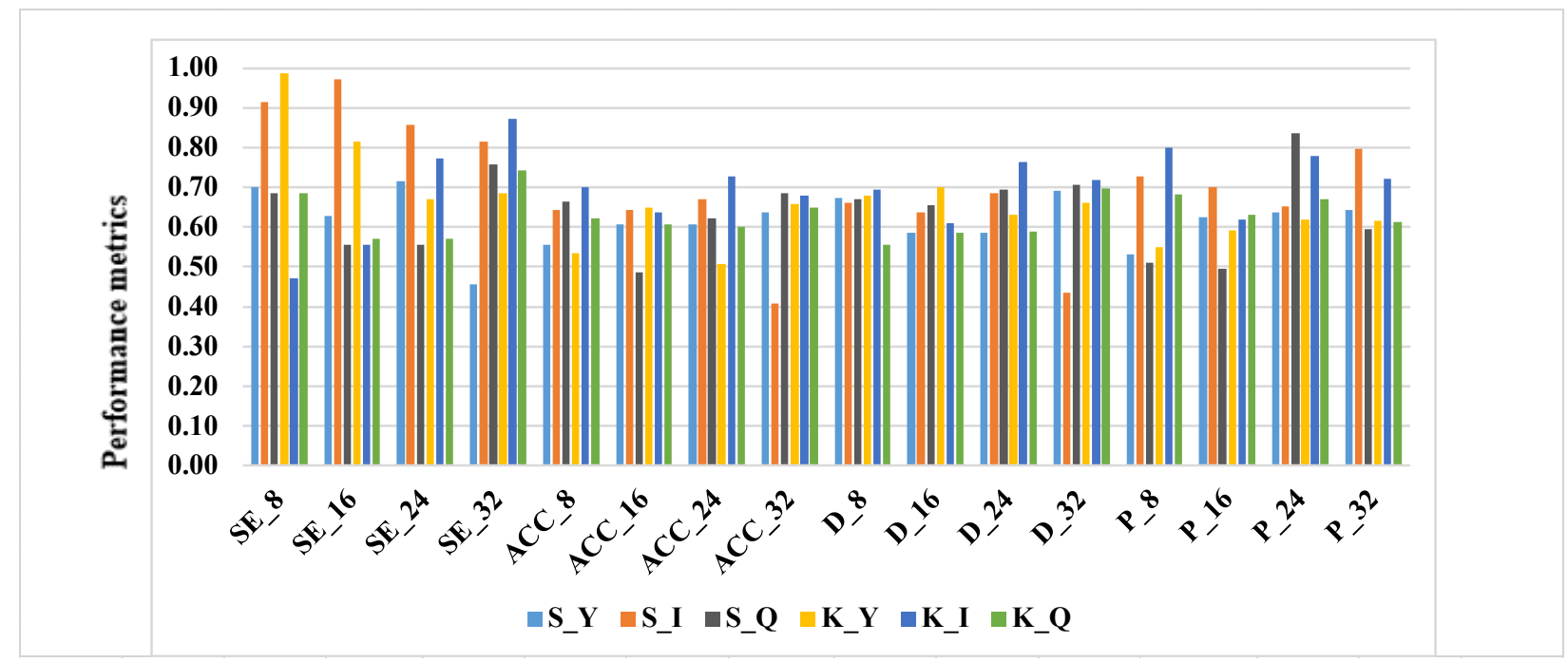

(b)

Fig. 5. Interpretation of the FFBPN performance based on sensitivity, accuracy,

Dice coefficient and precision.

(a) RGB channels; (b) YIQ channels. The following notations were used in the charts in Figures 5: - $S \_R, S \_G, S \_B$ are the skewness features computed for red, green and blue color channels; $\bullet K \_R$, $K \_\bar{G}, K_{-} \bar{B}$ are the kurtosis features computed for $r e d$, green and blue color channels; $\bullet S_{-} Y, S_{-} I, \bar{S} \_Q$ are the skewness features computed for luminance, I and $Q$ channels (in-phase and in-quadrature); • $K_{-} Y, K_{-} I, K \_Q$ are the kurtosis features computed for computed for luminance, $I$ and $Q$ channels (inphase and in-quadrature); $\bullet S E_{-} I, A_{C} C_{-} I, D_{-} I_{1} P_{-} I_{\text {are sensitivity, accuracy, Dice coefficient and }}$ precision for $\bar{I}=8, \overline{1}, \overline{2} 4,32$ hidden neurons.

According to the data in Figure 5, in the RGB space color, S_R (skewness features computed for red channel) and K_R (kurtosis features computed for red channel) provide the highest values of all the performance measures for the FFBPN classification and number of hidden neurons. The least accurate is K_G (kurtosis features computed for green channel). For the YIQ space, S_I (skewness features computed for I chromatic difference) and K_I (the kurtosis features computed for I channel - in-phase) provide the highest values for all performance measures for FFBPN classification and a number of hidden neurons. The least accurate is the S_Y metric (skewness features computed for luminance channel). 
The simplest and yet the most commonly used error function, whose purpose is to estimate the performance of neural networks is the mean square error (MSE). In table 1, the MSE values for each RGB and YIQ color space and for each number of hidden neurons are displayed.

Table1. Performance comparison of neural network with MSE for both color spaces RGB and YIQ

\begin{tabular}{|c|c|c|c|c|}
\hline \multirow{2}{*}{ Features } & \multicolumn{4}{|c|}{ Number of hidden neurons } \\
\cline { 2 - 5 } & 8 & 16 & 24 & 32 \\
\hline S_R & 0.222 & 0.190 & $\mathbf{0 . 0 5 4}$ & 0.171 \\
\hline S_G & 0.225 & 0.209 & $\mathbf{0 . 1 0 5}$ & 0.212 \\
\hline S_B & 0.250 & 0.454 & $\mathbf{0 . 1 5 4}$ & 0.412 \\
\hline K_R & $\mathbf{0 . 1 4 7}$ & $\mathbf{0 . 1 1 8}$ & $\mathbf{0 . 1 7 0}$ & $\mathbf{0 . 1 2 3}$ \\
\hline K_G & 0.237 & 0.222 & $\mathbf{0 . 1 0 3}$ & 0.217 \\
\hline K_B & 0.167 & 0.174 & $\mathbf{0 . 1 2 8}$ & 0.184 \\
\hline S_Y & 0.203 & 0.202 & $\mathbf{0 . 1 2 6}$ & 0.159 \\
\hline S_I & 0.210 & 0.202 & $\mathbf{0 . 1 2 9}$ & 0.161 \\
\hline S_Q & 0.209 & 0.194 & $\mathbf{0 . 1 9 6}$ & 0.178 \\
\hline K_Y & 0.215 & 0.200 & $\mathbf{0 . 1 9 5}$ & 0.189 \\
\hline K_I & 0.203 & 0.214 & $\mathbf{0 . 1 8 9}$ & 0.199 \\
\hline K_Q & 0.203 & 0.197 & $\mathbf{0 . 1 4 8}$ & 0.168 \\
\hline
\end{tabular}

A FFBNP architecture can successfully perform any task if it has enough neurons in the hidden layers. The best FFBNP architecture has the number of neurons in the hidden layer which gives the lowest MSE value for the training set. From the data in Table 1, it can be noticed that the lowest MSE value is achieved for the network model with 24 hidden neurons and for all investigated features. When FFBPN performance via MSE values is correlated to performance measures, the following conclusions are drawn in Table 2.

Table 2. Correlation between the FFBPN classification performance (for 24 hidden neurons) and performance measures (sensitivity SE, accuracy ACC, Dice coefficient D and precision P). The bold face represents the best values and the underlined are the second-best values.

\begin{tabular}{|llllll|}
\hline \multicolumn{6}{|c|}{ 24 hidden neurons in the hidden layer } \\
\hline & SE & ACC & D & P & MSE \\
\hline K_R & $\mathbf{0 . 9 7 1 4}$ & 0.6500 & 0.7243 & 0.5702 & 0.176 \\
\hline S_R & 0.7571 & $\mathbf{0 . 8 8 7 1}$ & $\mathbf{0 . 7 2 1 1}$ & $\mathbf{0 . 6 8 8 3}$ & $\mathbf{0 . 0 5 4}$ \\
\hline S_I & $\underline{0.8571}$ & $\underline{0.6714}$ & 0.6849 & 0.6522 & $\underline{0.129}$ \\
\hline K_I & 0.7714 & 0.7286 & 0.7625 & 0.7795 & 0.189 \\
\hline S_Q & 0.5571 & 0.6214 & 0.6936 & 0.8344 & 0.196 \\
\hline
\end{tabular}

According to the analysis given in Table 2, very good performance of the net's classification is determined for the S_R feature with an accuracy of $88.71 \%$ and MSE of 0.054 . Also, a good correlation between FFBPN classification performance (for 24 hidden neurons) and performance measures is provided by the S_I feature. It is also worth noting that the net's performance in the YIQ 
color space approach tends to be overestimated because the performance of YIQ in the skin lesions discrimination is quite low. Regarding the statistics features used in this analysis, skewness is seen to be worthy of investigation as a future direction of study.

\section{CONCLUSIONS}

This paper proposed a model which integrates an artificial neural network for the classification of malign and benign skin lesions, two color spaces (RGB and YIQ), and two statistics texture features. The obtained statistics texture features for each color channel were used as inputs for FFBPNs. The final architecture of FFBPN for classifications of skin lesions has 24 neurons in the hidden layer. This net is capable to achieve a classification accuracy of $88.71 \%$ and MSE of 0.054 for S_R in RGB color space and accuracy of $67.14 \%$ and MSE of 0.129 for K_I in YIQ color space, respectively. Our contribution is to design a net able to classify skin lesions more accurately. The results indicate S_R (skewness features computed for the red channel in RGB color space) as the best choice to reach the goal of our study. The reported result shows the advantages of monochrome channels representation for skin lesions diagnosis.

\section{References}

1. Ma J., Fan X., Yang S.X., Zhang X., Zhu X., Contrast Limited Adaptive Histogram Equalization Based Fusion for Underwater Image Enhancement, International Journal of Pattern Recognition and Artificial Intelligence 32(7) (2018) 1-27.

2. Rahman M. A, Haque M. T, Shahnaz C., Fattah S.A., Zhu W. P., Ahmed M. O., Skin lesions classification based on color plane-histogram-image quality analysis features extracted from digital images, in 2017 IEEE $60^{\text {th }}$ International Midwest Symposium on Circuits and Systems (MWSCAS), Medford, MA, United States, (2017) 1356-1359.

3. Kwok N., Ha Q., Fang G., Effect of color space on color image segmentation, In 2nd International Congress on Image and Signal Processing, Tianjin, China, (2009) 1-5.

4. Khan J., Malik A. S., Kamel N., Dass S. C., Affandi A. M., Segmentation of acne lesion using fuzzy C-means technique with intelligent selection of the desired cluster, In 37th Annual International Conference of the IEEE Engineering in Medicine and Biology Society (EMBC), Milan, Italy, (2015) 3077-3080.

5. Damian F. A., Moldovanu S., Moraru L., Colour histogram analysis for skin lesions discrimination, In $19^{\text {th }}$ International Multidisciplinary Scientific GeoConference: SGEM, Vienna, Austria, 19(6.3) (2019) 75-82.

6. Bakheet S., Al-Hamadi A., Computer-Aided Diagnosis of Malignant Melanoma Using GaborBased Entropic Features and Multilevel Neural Networks. Diagnostics 10(10) (2020) 822.

7. Kanimozhi T., Murthi A., Computer aided Melanoma skin cancer detection using Artificial Neural Network classifier, Singaporean Journal of Scientific Research (SJSR), Journal of Selected Areas in Microelectronics (JSAM) 8(2) (2016) 35-42.

8. Cui X., Niu Y., Zheng X., Han Y., An optimized digital watermarking algorithm in wavelet domain based on differential evolution for color image, PLoS One, 13(5) (2018) 1-15.

9. Doane D., Seward L., "Measuring Skewness: A Forgotten Statistic?", Journal of Statistics Education 19(2) (2011) 6-9.

10. Westfall P. H., Kurtosis as Peakedness, 1905 - 2014. R.I.P., The American Statistician 68(3) (2014) 191-195.

11. Kumar M., Alshehri M., AlGhamdi R., Sharma P., Deep V., A DE-ANN Inspired Skin Cancer Detection Approach Using Fuzzy C-Means Clustering, Mobile Networks and Applications 25(4) (2020) 1319-1329.

12. Ullah H, Kiber M. A., Huq A. H. M. A., Bhuiyan M. A. S., Performance Evaluation of Feed Forward Neural Network for Image Classification, Journal of Science and Technology 10(1) (2018) 19-27. 
13. Abdel-Ilah L., Šahinbegović H., Using machine learning tool in classification of breast cancer, Proceedings of the International Conference on Medical and Biological Engineering 62 (2017) 3-8.

14. Worring M., Gevers T., Interactive retrieval of color images, International Journal of Image and Graphics 1(03) (2001) 387-414.

15. Rajinikanth V., Couceiro M. S., RGB Histogram based Color Image Segmentation Using Firefly Algorithm, Procedia Computer Science 46 ( 2015 ) 1449 - 1457.

16. Thamizhvani T. R., Hemalatha R. J., Babu B., Dhivya A. J. A., Joseph J.E., Chandrasekaran R., Identification of Skin Tumours using Statistical and Histogram Based Features, Journal of Clinical and Diagnostic Research (JCDR) 12(9) (2018) 11-15. 